(n = 212); of these, $10(5 \%)$ participants were sensitised to mouse proteins. Prevalence of sensitisation was $3.5 \%$ in IVC only facilities and $6.3 \%$ in open cage/mixed facilities. Although numbers are small, a history of atopy to common aeroallergens and the site at which people worked appears to be associated with risk of sensitisation. Reporting of symptoms of laboratory animal allergy was as anticipated. (Table 1).

Conclusion The results from the SPIRAL study will be used to develop an evidence-based "Code of best working practices" for facilities using IVC systems, nationally and further afield, with the overall goal of significantly reducing the incidence of LAA.

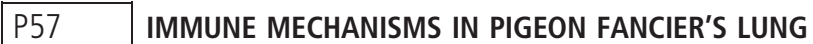

${ }^{1} S$ Hasan, ${ }^{2} \mathrm{~S}$ Bourke, ${ }^{1} \mathrm{~N}$ Kakkar, ${ }^{3} \mathrm{~A}$ Heaps, ${ }^{4} \mathrm{C}$ McSharry, ${ }^{1} \mathrm{~S}$ Todryk. ${ }^{1}$ Northumbria University, Newcastle Upon Tyne, UK; ${ }^{2}$ Royal Victoria Infirmary, Newcastle Upon Tyne, UK; ${ }^{3}$ Cumberland Infirmary, Carlisle, UK; ${ }^{4}$ Glasgow University, Glasgow, UK

\subsection{6/thoraxjnl-2015-207770.194}

Background Pigeon fanciers have a 1 in 10 risk of developing Pigeon Fancier's Lung (PFL), a form of hypersensitivity pneumonitis due to inhaled pigeon antigens. This manifests as acute episodes of breathlessness, cough and fever after antigen exposure, which may progress to chronic dyspnoea and lung fibrosis. The precise disease mechanisms are unclear but both antibody and $\mathrm{T}$ cell responses specific for pigeon antigens are implicated. We wish to better understand immune responses against pigeon antigens in PFL to give new insight into the disease mechanisms and provide disease markers.

Methods We studied the antibody and cellular immune response in fanciers attending pigeon shows. 77 completed a questionnaire of symptoms, performed spirometry and gave a blood sample, 32 of whom had symptoms of acute PFL. Peripheral Blood Mononuclear Cells and serum were extracted from the blood, and $\mathrm{T}$ cell and antibody responses were measured against pigeon serum and mucin using in-house well-characterised assays (ELISA and ELIspot, respectively), as well as flow cytometry and multiplex cytokine assays. These provided quantitative outputs of specific antibody titre and reactive $\mathrm{T}$ cells per million PBMC, validated using positive controls. Correlations between immune responses and disease symptoms were analysed.

Results All fanciers examined had significant antibody and $\mathrm{T}$ cell responses against pigeon serum and/or pigeon mucin antigen. A range of antibody avidities and isotypes were observed (Figure $1 ; \mathrm{n}=37 ; 2012$ cohort), but only $\operatorname{IgA}$ appeared to correlate with symptoms score $(\mathrm{p}=0.012)$. T cell responses to pigeon serum were measured in the form of proliferating cells positive for lung-homing receptors (alpha4beta1 integrin), and secreting gamma-interferon and large amounts of anti-inflammatory interleukin 10; but did not correlate with symptoms.

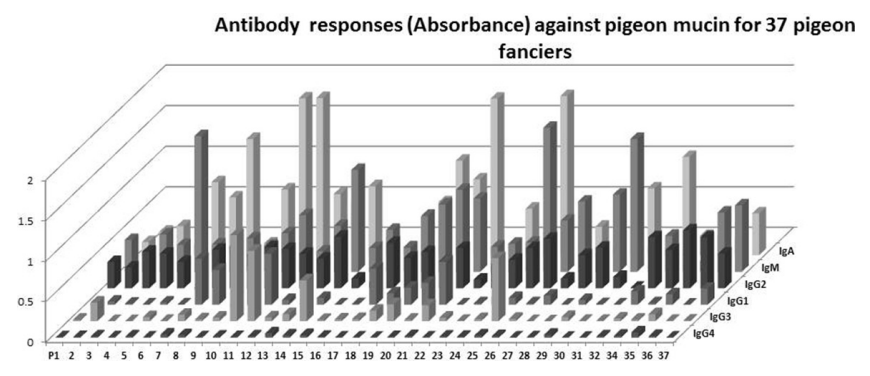

Abstract P57 Figure 1
Conclusions Pigeon fanciers have high levels of antigen exposure and demonstrate intense antibody and cellular immune responses, but these correlate minimally with clinical symptoms. The pathogenesis of PFL is complex and may involve an inflammatory cell-antibody axis.

\section{P58 CARRY OUT OF ANIMAL ALLERGENS FROM ANIMAL FACILITY ON SKIN OF LABORATORY ANIMAL WORKERS}

${ }^{1} \mathrm{H}$ Campbell, ${ }^{1} \mathrm{~J}$ Canizales, ${ }^{2} \mathrm{~S}$ Semple, ${ }^{1} \mathrm{~J}$ Feary, ${ }^{1} \mathrm{P}$ Cullinan, ${ }^{1} \mathrm{M}$ Jones. ${ }^{1}$ Imperial College, London, UK; ${ }^{2}$ Aberdeen University, Aberdeen, UK

\subsection{6/thoraxjnl-2015-207770.195}

Introduction There are at least 12,000 laboratory animal workers in the UK who are at risk of developing an IgE-associated respiratory allergy to airborne animal proteins. There has been a drive to reduce animal allergen levels in animal facilities, however recent studies have suggested that laboratory workers may also transfer animal allergens outside of the animal facility to their offices, laboratories and indeed their homes. Among Scottish technicians Mus m 1 was detected on hands, shoes, car steering wheels and domestic door handles after leaving work (S. Semple - personal communication). Krop et al. detected significantly higher levels of mouse allergen in mattresses from the homes of laboratory animal workers than those from nonexposed controls suggesting carry out of allergen from work to home. These observations may have significant clinical relevance; in Poland, children of laboratory animal workers had a higher prevalence of sensitisation to mouse than did the children of parents in other occupations.

The aim of our study was to evaluate whether mouse allergen is transferred out of the animal facility on the skin of workers.

Methods We examined dermal wipes taken from forehead, palms and back of hands of both laboratory animal workers and non-exposed workers, when they entered the facility in the morning and again when they were ready to depart at the end of the day. Mus $\mathrm{m} 1$ was extracted from the wipes and levels quantified using an ELISA (Indoor Biotechnology).

Results Mus $\mathrm{m} 1$ levels were significantly higher in the dermal wipes taken at the end of the day compared to those taken in the morning $(\mathrm{p}<0.05)$, in the laboratory animal workers but not in the non-exposed controls (Figure 1).

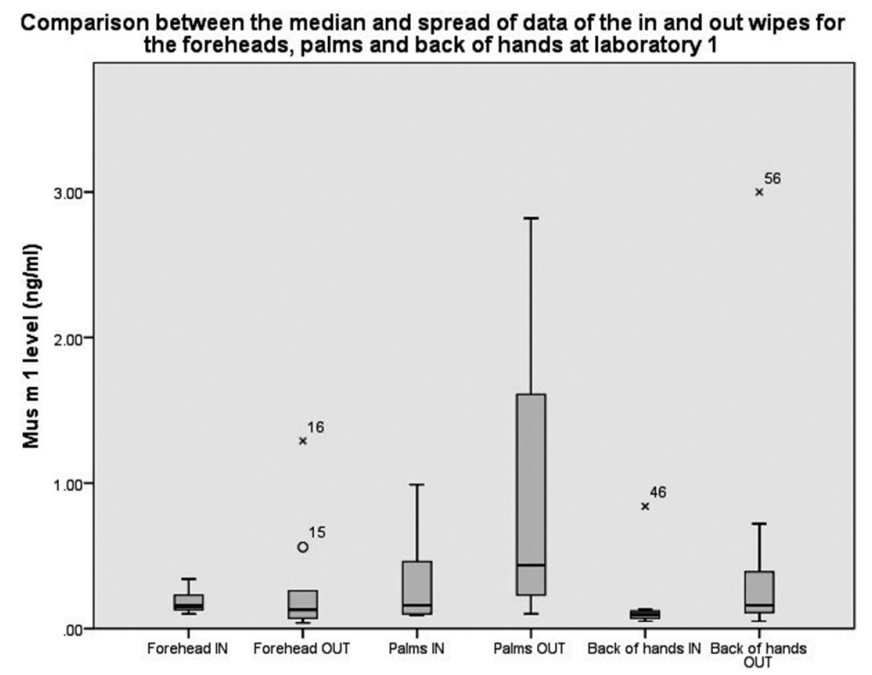

Abstract P58 Figure 1 